\title{
Stability of Platelet-rich Fibrin Treated with Tranexamic Acid In Vivo: A Histological Study in Rats
}

\author{
Vellayappan Radha ${ }^{1}$, Sheeja Saji Varghese ${ }^{2}$, Mohanraj Karthik Ganesh ${ }^{3}$
}

\begin{abstract}
Aim and objective: The present study aimed to evaluate the effect of tranexamic acid on the stability of platelet-rich fibrin (PRF) and its influence on connective tissue healing in Wistar rats.

Materials and methods: This experimental study included 12 male Wistar rats in three groups; group I-control (saline), group II-untreated PRF, and group III-PRF treated with tranexamic acid (PRF + TXA). Platelet-rich fibrin membrane was prepared by drawing blood from each rat and the prepared membrane was cut into two halves wherein one half was treated with tranexamic acid. After anesthetizing the animal, a linear incision was made on the mid-posterior part of the dorsal side of the body to expose the muscle and three sites were prepared. Sterile saline was applied in the control site, untreated PRF membrane was placed in group II site, and PRF + TXA in group III site. Six rats were sacrificed at 14 days and the other six at 28 days. Specimens were subjected to histological evaluation for measuring the remaining PRF and immunohistochemical analysis for evaluating vimentin expression for assessing connective tissue healing.

Results: Histopathological evaluation revealed that group III had more area of aggregation of PRF bundles at 28 days than group II. On immunohistochemical imaging, the intensity and proportionality score of vimentin expression was more in group III at 28 days.

Conclusion: From the study, it can be concluded that tranexamic acid is effective in delaying the degradation of the PRF membrane and has a positive influence on connective tissue healing.

Clinical significance: Improved stability of PRF observed with the addition of tranexamic acid will have wider applications in regenerative therapy using PRF.

Keywords: Healing, Innovation, Platelet-rich fibrin, Regeneration, Tranexamic acid, Wistar rats.

World Journal of Dentistry (2021): 10.5005/jp-journals-10015-1861
\end{abstract}

\section{INTRODUCTION}

Periodontitis is a common inflammatory destructive disease affecting the supporting structures of the teeth resulting in tooth mobility and tooth loss. The goals of periodontal treatments are to control the inflammation and facilitate a clinical environment favorable for regaining the lost periodontal support. ${ }^{1}$ The main aim of periodontal therapy is to regenerate all the lost tissues of the periodontium and establish a new attachment on a root surface affected by periodontal disease. The process of new attachment involves many cellular events such as cell migration, cellular adhesion, cellular proliferation, and differentiation in a well-orchestrated manner. This complex process is controlled by many inflammatory cytokines, morphogens, and growth factors. ${ }^{2}$ Evidence suggests that platelets play a crucial role in inflammation and periodontal wound healing by providing cytokines and growth factors. ${ }^{3}$ Various regenerative therapies currently in clinical practice, including bone grafting, guided are showing variable results in healing and periodontal regeneration.

Choukroun et al. ${ }^{4}$ were the first one who described a new platelet concentrate namely platelet-rich fibrin (PRF), and proposed the protocol for its preparation. Platelet-rich fibrin is considered as the second-generation of platelet concentrates and structurally resembles a membrane made of fibrin. It is prepared from the patient's own blood without using any anticoagulants. The PRF consists of a natural fibrin matrix wherein platelets and growth factors are found in high concentration., 4 Platelet-rich fibrin membrane is considered as a versatile regenerative material not only due to its structural framework, which can act as a scaffold during healing, but also due to the presence of numerous growth
1,2Department of Periodontics, Saveetha Dental College and Hospitals, Saveetha Institute of Medical and Technical Sciences, Chennai, Tamil Nadu, India

${ }^{3}$ Department of Anatomy, Biomedical Research Unit and Lab Animal Centre, Saveetha Dental College and Hospitals, Saveetha Institute of Medical and Technical Sciences, Chennai, Tamil Nadu, India

Corresponding Author: Sheeja Saji Varghese, Department of Periodontics, Saveetha Dental College and Hospitals, Saveetha Institute of Medical and Technical Sciences, Chennai, India, Phone: +91 9884042252, e-mail: drsheeja@rediffmail.com

How to cite this article: Radha V, Varghese SS, Ganesh MK. Stability of Platelet-rich Fibrin Treated with Tranexamic Acid In Vivo: A Histological Study in Rats. World J Dent 2021;12(5):386-391.

Source of support: Saveetha Institute of Medical and Technical Science Conflict of interest: None

factors and matrix cellular proteins including platelet-derived growth factor, transforming growth factor, vascular endothelial growth factor, insulin-like growth factor, and thrombospondin-1, which can enhance the healing and regeneration. Also, the complex architecture of PRF acts as a scaffold with distinctive biomechanical characteristics, which makes it unique in comparison with other platelet concentrates.

In an in vitro cell culture study using periodontal ligament fibroblasts demonstrated that alkaline phosphatase activity, extracellular signal-regulated protein kinase phosphorylation, and osteoprotegerin expression were stimulated by PRF. ${ }^{6}$ In a clinical trial done in periodontitis patients, it has been observed that the 
combination of PRF with the commercially available GTR membrane was more effective in improving clinical and radiographic outcomes than the GTR membrane alone in the management of osseous defects. ${ }^{7}$ Platelet-rich fibrin was demonstrated to have continual release of growth factors and have less susceptibility to degradation by proteolytic enzymes after 24 hours in comparison with other agents like recombinant platelet-derived growth factor isoforms, thrombin-activated platelet concentrates, and autologous fibrin sealant in an in vitro study. ${ }^{8}$

Despite its biological merits, one of the biggest challenges in using PRF over other barrier membranes is its rapid degradation. Sam et al. $^{9}$ have reported that in comparison with other commercially available barrier membranes, PRF had a higher degradability and lesser rigidity. Also stated that, due to this PRF gets resorbed faster and gets collapsed into the periodontal defect which makes it unsuitable for maintaining the space in guided tissue regeneration. A study where the stability of PRF was assessed both histologically and immunohistochemically in rat models reported that the PRF became thin at 21 days, and completely disappeared at 28 days. ${ }^{10}$ These results emphasize that though PRF can have a stimulatory effect on tissue regeneration and healing, its faster degradation might be a hindering factor while using clinically. This also points out that PRF cannot be used as a barrier membrane for the space maintenance in periodontal regeneration.

Tranexamic acid, a synthetic derivative of the amino acid lysine, is an anti-fibrinolytic agent acting by blocking the lysine binding sites on plasminogen molecules. It is used in many bleeding disorders to reduce postoperative bleeding and transfusion requirements. Also, tranexamic is used as a potent alternative to surgery in menorrhagia due to its ability to reduce menstrual blood loss. It is also more effective than any other synthetic antifibrinolytic agent due to its binding to plasminogen. ${ }^{11,12}$

It has been observed that one preoperative intravenous injection of tranexamic acid could prevent excessive bleeding during extraction of third molars. ${ }^{11-13}$ Similarly, in patients with bleeding disorders, administration of tranexamic acid for 5 days could significantly reduce the excessive bleeding during dental extractions. ${ }^{14}$ Moreover, there were no side effects and toxic reactions observed in heart, kidney, or liver during the course of the drug administration. ${ }^{14}$ Based on these observation, we hypothesized that adding tranexamic acid to PRF might delay its degradation and a preliminary in vitro experiment done by our team observed that tranexamic acid had a promising effect on delaying the degradation of PRF membrane at various concentrations used and there was no significant difference between the different concentrations tested $(50-200 \mathrm{mg} / \mathrm{mL}) .^{15}$ These results propose the potential of tranexamic acid in improving the stability of PRF, thereby improving its therapeutic potential in regeneration and healing. So far no in vivo research has been done in this direction validating the effectiveness of tranexamic acid on the stability of PRF and its influence on healing. Hence, the present study was aimed to examine the stability of tranexamic acid-treated PRF and compare the same with untreated PRF in vivo in rat models at 14 and 28 days. The study also aimed to evaluate its effect on tissue healing by immunohistochemical evaluation.

\section{Materials and Methods}

This in vivo animal study was done at Biomedical Research Unit and Lab Animal Centre (BRULAC), Saveetha Dental College and
Hospitals. The research protocol was approved by the Institutional Animal Ethical Committee and Review Board (BRULAC/SDCH/ SIMATS/IAEC/02-2019/020). Twelve male Wistar rats, 15 weeks old, with an average weight of $300 \mathrm{~g}$ were used in this study. Animals were procured from the Center for Laboratory Animal Research (CLAR), Saveetha Institute of Medical and Technical Science (Reg. No: 865/04/CPCSEA Dt; 16/12/04). Anesthesia was achieved with a combination of ketamine $(40 \mathrm{mg} / \mathrm{kg}$ ) and xylazine $(5 \mathrm{mg} / \mathrm{kg})$. The PRF membrane was prepared in accordance to the Choukroun method. ${ }^{4}$ Approximately $1 \mathrm{~mL}$ of blood was collected from the superficial femoral vein with a small syringe and a needle, later transferred to a $1 \mathrm{~mL}$ Durham glass tube (Maruemu Durham $8 \mathrm{~L}$, Japan) and immediately centrifuged for 10 minutes at 12,000 RPM. After centrifugation, three layers were formed: supernatant on the top, PRF gel in the middle, and red blood cell layer at the bottom. Platelet-rich fibrin gel was collected after cutting between the junction of the middle layer and the RBC layer, including the small top portion of the RBC layer. The clot was placed inside gauze and pressed and the PRF membrane was obtained. The prepared PRF membrane was cut into two halves and one half was treated with tranexamic acid. Commercially available tranexamic acid ampoule (tramy-Z $500 \mathrm{mg} / 5 \mathrm{~mL}$ ) was used for treating PRF for 1 hour. After, shaving and disinfecting the dorsum of rat (the surgical area) with a solution of iodine and $70 \%$ alcohol, a linear incision on the mid-posterior part of the body of the rat was performed and the muscle was exposed. Considering three groups, group I-control, group II-untreated PRF, and group III-PRF treated with tranexamic acid (PRF + TXA), two small incisions $(1.5 \mathrm{~mm}$ ) on the left side and one incision $(1.5 \mathrm{~mm})$ on the right side were made on the surface of the muscle of the mid-posterior part of every rat. The three incisions were minimum $2 \mathrm{~mm}$ apart. On the left side top incision, sterile saline was applied and the muscle was sutured again. This served as the control. On the left lower incision, the untreated PRF membrane was placed into the incised muscle and sutured. On the right side, the third incision, PRF + TXA was placed into the muscle and sutured. Braided silk 4-0 sutures were used to secure the PRF membrane and this non-resorbable suture was served as a guide to harvest the tissue later. The rats were fed with standard laboratory food and water ad libitum during the course of the study. Six rats were sacrificed at 14 days and other six at 28 days by inhalation of isoflurane in excess. A $10 \times 10 \mathrm{~mm}$ portion of tissue from the surrounding area was excised. The tissue specimens were fixed in a solution of $10 \%$ neutral buffered formalin for 14 days, dehydrated, and then embedded in paraffin. The paraffin mold was then sliced in $3 \mu \mathrm{m}$ sections and stained using routine hematoxylin-eosin. The specimen was then observed under an optical microscope (Biozero, Keyence, Tokyo, Japan). The images from the histological samples were captured. The area of PRF was measured with an image analyzer 21 (Image J., National Institute of Health, USA).

\section{Immunohistochemistry}

To assess the fibroblast population vimentin was used as an immunohistochemical marker. For immunohistochemical evaluation, the slides were prepared from the formalin-fixed paraffin-embedded blocks. Three micrometer thick sections were cut from the block and were mounted on gelatin-coated slides. Deparaffinization of the sections was done using xylene followed by dehydration by alcohol and finally rinsing with distilled water. For retrieval of antigen, heat induced-epitope retrieval in citrate buffer (pH 6.0) was used for 10 minutes in a pressure cooker. Following 
which endogenous peroxidase was blocked using peroxide block for 5 minutes followed by protein block for 5 minutes. Sections were then incubated with primary antibody vimentin (Dako, Monoclonal Mouse Anti-vimentin, Clone V9) and incubated for 60 minutes. Novolink Min Polymer Detection System (Leica Microsystems, Newcastle, UK) was used for the detection of antigen antibody complexes. For this the sections were counterstained with Mayer's hematoxylin and were dehydrated and mounted. Both positive and negative controls were used for the comparison. Fibro sarcoma sections were used as a positive control for vimentin. Negative controls were made by staining the slides without using the primary antibody.

\section{Evaluation of Slides}

The presence of a brown-colored end product at the site of the target antigen was indicative of positive reactivity. Stromal spindle cells that were positive for vimentin were regarded as fibroblasts. The staining intensity was graded from 0 to 3 (negative, weak, moderate, and intense). The proportionality score was graded as 0-2 (negative, focal, and diffuse). The total score of each sample was calculated as the sum of intensity score and the proportionality score. For the immunohistochemical evaluation, stained sections were evaluated by two independent observers and inter-examiner variability was assessed with $\mathrm{K}$ statistics. Statistical analysis was done by SPSS software version 20 .

\section{Results}

In the histological images, the transplanted PRF, which showed bluish color with a characteristic structure, was distinguishable from the surrounding tissue (Figs $1 B$ and G). Notably, with time, the transplanted PRF became small in both the groups (group II and III) (Figs $1 \mathrm{H}$ and I). At 14 days, intact epidermal and dermal area with connective tissue and underlying muscle fasciculi with mild scar tissue of the surgical site was seen. Below the subcutaneous tissue, the PRF bundles as aggregation of floccules with intense eosinophilic stained areas were seen at the implanted surgical site. Underneath the subcutaneous tissue the PRF + TA group (Figs 1F and I) showed increased aggregation of PRF bundles as floccules with intense eosinophilic stained areas. And at 28 days, group II (untreated PRF) (Fig. 1H) showed degeneration of PRF bundles with reduced floccules at the implanted surgical site and group III (PRF + TA) (Fig. 1I) showed comparatively more aggregation of PRF bundles with retention of PRF floccules at the implanted surgical site than PRF group. The area of PRF was measured with an image analyzer and was statistically compared. The statistical test performed was independent $t$-test to compare the area of PRF between the groups at 14 and 28 days (Table 1). In group II (untreated PRF), the mean area of PRF was $5.06 \pm 0.46 \mathrm{~mm}^{2}$ at 14 days and $1.11 \pm 0.35$ at 28 days. In group III (PRF + TXA), the mean area of PRF was $5.55 \pm 0.40$ $\mathrm{mm}^{2}$ at 14 days and $1.92 \pm 0.31$ at 28 days. However when mean area of PRF was compared between the groups, PRF + TXA showed significantly larger area of PRF at $14(p=0.01)$ and 28 days $(p=0.00)$ when compared with untreated PRF group (Table 1).

Immunohistochemical analysis showed that the mean total score is highest in group III (3.5 \pm 0.22$)$ followed by group II ( 2.75 $\pm 0.75)$ and least in group I (1.5 \pm 1.6$)$ (Table 2). To calibrate, interexaminer agreement, Cohen's $\kappa$ statistics was done using SPSS. For both control group (group I) and untreated PRF group (group II), Cohen's $\kappa$ was 0.86 and for PRF + TXA group (group III), Cohen's $\kappa$ was 0.83 which reflects a good strength of agreement.

\section{Discussion}

The present study was aimed to evaluate whether treating PRF membrane with tranexamic acid would delay the degradation of PRF membrane and have any influence on tissue healing in Wister rat models. On histopathological evaluation done on 14th and 28th day, it was observed that PRF + TXA showed significantly larger areas of aggregation of remaining PRF bundles when compared with untreated PRF group. On immunohistochemical imaging, the intensity and proportionality score of vimentin expression of fibroblast during the healing was more in PRF + TXA group at 28 days when compared with other two groups.

The larger area of remaining PRF in the tranexamic acidtreated PRF group denotes that the tranexamic acid delayed the degradation of PRF in vivo. An in vitro study done by our group supports this finding wherein tranexamic acid delayed the disintegration of PRF at various concentrations. ${ }^{15}$ Delayed disintegration of PRF could be due to the anti-fibrinolytic effect of tranexamic acid which reversibly blocks the lysine binding sites on plasminogen molecules. ${ }^{11}$

In the present study, vimentin expression was evaluated immunohistochemically to know whether addition of tranexamic acid on PRF had any influence on tissue healing. Vimentin was used to evaluate tissue healing as it is the major structural component of mesenchymal cells such as fibroblasts and endothelial cells. ${ }^{16}$ Results revealed that the vimentin expression was higher in both the PRF groups when compared with control and highest in the tranexamic acid-treated PRF group. This results support the view that adding PRF into the surgical site improves the healing and nevertheless the addition of tranexamic acid has a positive impact on healing. Increase in vimentin expression was observed till 28th day. This may signify that the stimulatory effect of PRF through the release of growth factors may extend till 28 days. It has been reported that addition of tranexamic acid in the extracted sockets shortened the duration of wound healing following extractions in rabbits. ${ }^{17}$

Numerous studies ${ }^{18-22}$ have confirmed the usefulness of PRF in the management of periodontal defects. In the management of gingival recession with coronally advanced flap, the adjunctive use of PRF membrane was found to have increased the keratinized gingival width when compared with coronally advanced flap alone. ${ }^{23,24}$ Tranexamic acid has a wide range of clinical applications ranging from as an anti-fibrinolytic agent during knee and hip arthroplasty to as a mouth rinse to reduce bleeding during oral surgical procedures. It is also widely used in reducing menstrual blood loss and reducing the risk of thromboembolic complications in pregnancy.

The degradability of PRF was evaluated by both in vitro and in vivo studies. On comparing the degradability of PRF with other commercially available membranes in vitro, up to 6 days PRF membrane was able to maintain a comparable physical property in comparison with other commercially available membranes whereas after 1 week, the degradability was significantly higher than the other membranes. ${ }^{9}$ Also, an in vivo study using Wistar rat models reported that PRF completely disappeared in 28 days. ${ }^{10} \mathrm{An}$ in vitro study done by our team ${ }^{15}$ demonstrated that treating PRF with tranexamic acid significantly reduced the degradability of PRF after 1 week observation. The delayed disintegration of tranexamic acid-treated PRF can be attributed to the anti-fibrinolytic action of tranexamic acid on PRF. Tranexamic acid binds with the plasminogen through the high affinity lysine binding sites and 




Figs 1 A to I: Histopathology H\&E staining: (A) Group I control at 10× magnification (14 days); (B) Group II at 10× magnification (14 days); (C)

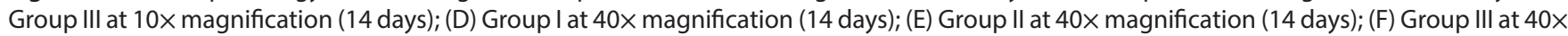
magnification (14 days); (G) Group I at 40× magnification (28 days); (H) Group II at 40× magnification (28 days); (I) Group III at 40× magnification (28 days)

Table 1: Histological measurement of PRF at various intervals between the groups

\begin{tabular}{lll}
\hline & 14th day & 28th day \\
\cline { 2 - 3 } & Mean $\pm S D$ & Mean $\pm S D$ \\
\hline Group II & $5.067 \pm 0.0 .47$ & $1.11 \pm 0.35$ \\
Group III & $5.5 \pm 0.4$ & $1.92 \pm 0.32$ \\
$\begin{array}{l}p \text { value (independent } \\
t \text {-test) }\end{array}$ & 0.013 & 0.000 \\
\hline
\end{tabular}

Table 2: Immunohistochemical scoring of vimentin expression of three groups

\begin{tabular}{lllll}
\hline & & $\begin{array}{l}\text { Group lat } \\
\text { 28 days }\end{array}$ & $\begin{array}{l}\text { Group II at } \\
\text { 28 days }\end{array}$ & $\begin{array}{l}\text { Group III at } \\
\text { 28 days }\end{array}$ \\
\hline $\begin{array}{l}\text { Total score } \\
(\text { Mean } \pm \text { SD) }\end{array}$ & Examiner 1 & $1.5 \pm 1.67$ & $2.75 \pm 0.75$ & $3.5 \pm 0.22$ \\
& Examiner 2 & $1.66 \pm 1.07$ & $2.83 \pm 0.71$ & $3.25 \pm 0.45$ \\
$\begin{array}{l}\text { Cohen's } \kappa \\
\text { value }\end{array}$ & 0.86 & 0.86 & 0.83 \\
\hline
\end{tabular}

thereby completely blocking the interaction of plasminogen and the plasmin with the lysine residues of fibrin monomer. ${ }^{12}$ Due to this process, the binding of plasminogen to the surface of fibrin is prevented and thereby impedes fibrinolysis. ${ }^{12}$

Multiple animal species such as rodents, rabbits, dogs, pigs, and non-human primates have been used in periodontal research.
A major drawback of using smaller animals when compared with primates is the limited similarity of their dentition to human dentition. However, small animals like mice and rats are very useful in periodontal research dealing with inflammation and tissue healing. ${ }^{25}$ In the present study, we used Wistar rats for the experiment as the study was intended to see the degradability of PRF and the soft tissue healing response. For establishing mammalian standards in laboratory settings, Wistar rats are considered as the ideal standardized mammal. ${ }^{26}$ Even though the rat cranium is the ideal site for testing the bone regenerative materials, ${ }^{27}$ in the present study, we have chosen the dorsal part of the rat as the experimental site as it is the common and preferred site for research related to soft tissue healing. ${ }^{28}$

In the present study, we did not evaluate whether tranexamic acid has any influence on the release of growth factors from PRF or has any direct interactions with the released growth factors. But this study has confirmed that tranexamic acid has a positive effect on delaying the degradation of PRF in vivo animal models. As an indigenous material platelet concentrates, including PRF has wider applications in tissue regeneration and healing including periodontal regeneration. ${ }^{21,22,29-31}$ In this context, the results of the present study, revealing the positive effect of tranexamic acid on PRF, is clinically significant and require further validation by clinical research. Some limitations of this study that need to be considered while interpreting the results are that it was only an animal study and the healing was evaluated not in a periodontal 
wound. Thus, before confirming the clinical efficiency of PRF in tissue healing and regeneration, further studies are required to check whether tranexamic acid has any influence on the release of growth factors from PRF. Moreover, to confirm its application in periodontal regeneration, further research needs to be carried out in experimentally induced periodontitis lesions in animal models before proceeding to clinical trials.

\section{Conclusion}

Tranexamic acid is effective in delaying the degradation of the PRF membrane and has a positive impact on connective tissue healing in rat models.

\section{Clinical Significance}

Improved stability of PRF observed with the addition of tranexamic acid will have wider applications in regenerative therapy using PRF.

\section{ACKnOWLedgments}

The authors are grateful to Dr Deepak Nallaswami, Director SIMATS for his guidance. The authors acknowledge Dr Herald J Sherlin, former professor, department of Oral and Maxillofacial Pathology, Saveetha Dental College and hospital for the help rendered for Histological and immunohistochemical analysis. There is no financial support for this study.

\section{Author Contribution}

All the authors have contributed to study inception, data interpretation, data analysis, and manuscript preparation.

\section{References}

1. Lang NP, Tonetti MS. Periodontal risk assessment (PRA) for patients in supportive periodontal therapy (SPT). Oral Health Prev Dent 2003;1(1):7-16.

2. Raja S, Byakod G, Pudakalkatti P. Growth factors in periodontal regeneration. Int J Dent Hyg 2009;7(2):82-89. DOI: 10.1111/j.16015037.2009.00380.x.

3. Giannobile WV. Periodontal tissue engineering by growth factors. Bone 1996;19(1 Suppl):S23-S37. DOI: 10.1016/s8756-3282(96)00127-5.

4. Choukroun J, Simonpieri A, Girard M-O, et al. Platelet rich fibrin (PRF): un nouveau biomatériau de cicatrisation. Implantodontie 2004;13(4):229-235. DOI: 10.1016/j.implan.2004.07.002.

5. Dohan DM, Choukroun J, Diss A, et al. Platelet-rich fibrin (PRF): a second-generation platelet concentrate. Part III: leucocyte activation: a new feature for platelet concentrates? Oral Surg Oral Med Oral Pathol Oral Radiol Endod 2006;101(3):e51-e55. DOI: 10.1016/j. tripleo.2005.07.010.

6. Chang $\mathrm{Y}-\mathrm{C}$, Zhao J-H. Effects of platelet-rich fibrin on human periodontal ligament fibroblasts and application for periodontal infrabony defects. Aust Dent J 2011;56(4):365-371. DOI: 10.1111/j.18347819.2011.01362.x.

7. Panda S, Sankari M, Satpathy A, et al. Adjunctive effect of autologus platelet-rich fibrin to barrier membrane in the treatment of periodontal intrabony defects. J Craniofac Surg 2016;27(3):691-696. DOI: $10.1097 /$ SCS.0000000000002524.

8. Lundquist R, Dziegiel MH, Agren MS. Bioactivity and stability of endogenous fibrogenic factors in platelet-rich fibrin. Wound Repair Regen 2008;16(3):356-363. DOI: 10.1111/j.1524-475X.2007.00344.x.

9. Sam G, Vadakkekuttical RJ, Amol NV. In vitro evaluation of mechanical properties of platelet-rich fibrin membrane and scanning electron microscopic examination of its surface characteristics. J Indian Soc Periodontol 2015;19(1):32-36. DOI: 10.4103/0972-124X.145821.
10. Yamashita $Y$, Chen Kang, Kuroda S, et al. Stability of platelet rich fibrin in vivo: histological study in rats. J Oral Tissue Engin 2016;14:83-90.

11. Svanberg L, Astedt B, Nilsson IM. Abruptio placentae--treatment with the fibrinolytic inhibitor tranexamic acid. Acta Obstet Gynecol Scand 1980;59(2):127-130. DOI: 10.3109/00016348009154628.

12. Dunn CJ, Goa KL. Tranexamic acid. Drugs 1999;57(6):1005-1032. DOI: 10.2165/00003495-199957060-00017.

13. Senghore N, Harris M. The effect of tranexamic acid (cyclokapron) on blood loss after third molar extraction under a day case general anaesthetic. Br Dent J 1999;186(12):634-636. DOI: 10.1038/ sj.bdj.4800183.

14. Forbes CD, Barr RD, Reid G, et al. Tranexamic acid in control of haemorrhage after dental extraction in haemophilia and Christmas disease. Br Med J 1972;2(5809):311-313. DOI: 10.1136/bmj.2.5809.311.

15. Radha V, Varghese SS. In vitro analysis to analyse the disintegration property of PRF when treated with various concentration of tranexamic acid. Int J Pharmaceut Sci Res 2018;9:4912-4916.

16. Franke WW, Hergt M, Grund C. Rearrangement of the vimentin cytoskeleton during adipose conversion: formation of an intermediate filament cage around lipid globules. Cell 1987;49(1):131-141. DOI: 10.1016/0092-8674(87)90763-x.

17. Vinckier F, Vermylen J. Blood loss following dental extractions in anticoagulated rabbits: effects of tranexamic acid and socket packing. Oral Surg Oral Med Oral Pathol 1985;59(1):2-5. DOI: 10.1016/00304220(85)90105-7.

18. Lekovic V, Milinkovic I, Aleksic Z, et al. Platelet-rich fibrin and bovine porous bone mineral vs. platelet-rich fibrin in the treatment of intrabony periodontal defects. J Periodontal Res 2012;47(4):409-417. DOI: 10.1111/j.1600-0765.2011.01446.x.

19. Sharma A, Pradeep AR. Treatment of 3-wall intrabony defects in patients with chronic periodontitis with autologous plateletrich fibrin: a randomized controlled clinical trial. J Periodontol 2011;82(12):1705-1712. DOI: 10.1902/jop.2011.110075.

20. Joseph VR, Rosamma Joseph V, Raghunath A, et al. Clinical effectiveness of autologous platelet rich fibrin in the management of infrabony periodontal defects. Singapore Dent J 2012;33(1):5-12. DOI: 10.1016/j.sdj.2012.10.003.

21. Panda S, Jayakumar N, Sankari M, et al. Platelet rich fibrin and xenograft in treatment of intrabony defect. Contemp Clin Dent 2014;5(4):550-554. DOI: 10.4103/0976-237X.142830.

22. Panda S, Doraiswamy J, Malaiappan S, et al. Additive effect of autologous platelet concentrates in treatment of intrabony defects: a systematic review and meta-analysis. J Investig Clin Dent 2016;7(1):1326. DOI: 10.1111/jicd.12117.

23. Aroca S, Keglevich T, Barbieri B, et al. Clinical evaluation of a modified coronally advanced flap alone or in combination with a platelet-rich fibrin membrane for the treatment of adjacent multiple gingival recessions: a 6-month study. J Periodontol 2009;80(2):244-252. DOI: 10.1902/jop.2009.080253.

24. Padma R, Shilpa A, Kumar PA, et al. A split mouth randomized controlled study to evaluate the adjunctive effect of platelet-rich fibrin to coronally advanced flap in Miller's class-I and II recession defects. J Indian Soc Periodontol 2013;17(5):631-636. DOI: 10.4103/0972-124X.119281.

25. Kantarci A, Hasturk $H$, Van, et al. Animal models for periodontal regeneration and peri-implant responses. Periodontol 2000 2015;68(1):66-82. DOI: 10.1111/prd.12052.

26. Clause BT. The Wistar rat as a right choice: establishing mammalian standards and ideal of a standardised mammal. J Hist Biol 1993;26(2):329-349. DOI: 10.1007/BF01061973.

27. Graves DT, Fine D, Teng Y-TA, et al. The use of rodent models to investigate host-bacteria interactions related to periodontal diseases. J Clin Periodontol 2008;35(2):89-105. DOI: 10.1111/j.1600051X.2007.01172.x.

28. Dorsett-Martin WA, Wysocki AB. Rat models of skin wound healing. Sourcebook Mod Biomed Res, 631-638.

29. Del Fabbro M, Karanxha L, Panda S, et al. Autologous platelet concentrates for treating periodontal infrabony defects. Cochrane 
Database Syst Rev 2018;11:CD011423. DOI: 10.1002/14651858. CD011423.pub2.

30. Panda S, Ramamoorthi S, Jayakumar ND, et al. Platelet rich fibrin and alloplast in the treatment of intrabony defect. J Pharm Bioallied Sci 2014;6(2):127-131. DOI: 10.4103/0975-7406.129178.
31. Ravi S, Malaiappan S, Varghese $S$, et al. Additive effect of plasma rich in growth factors with guided tissue regeneration in treatment of intrabony defects in patients with chronic periodontitis: a split-mouth randomized controlled clinical trial. J Periodontol 2017;88(9):839-845. DOI: 10.1902/jop.2017.160824. 